\title{
Fusobacterium nucleatum
}

National Cancer Institute

\section{Source}

National Cancer Institute. Fusobacterium nucleatum. NCI Thesaurus. Code C86404.

A species of anaerobic, Gram negative, fusiform rod shaped bacterium in the phylum Fusobacteria. This species is non-sporulating, ferments fructose and glutamate and produces butyric acid as a major metabolic by-product. F. nucleatum is ubiquitously found in the normal flora of the oral cavity but may be associated with periodontal disease as well as preterm births, where isolates have been found in amniotic fluid, placenta, and chorioamnionic membranes of women delivering prematurely. 